\title{
CRAQUEAMENTO TERMOCATALÍTICO DE POLIETILENO (PEBD E PEAD) EM ESCALA DE LABORATÓRIO
}

\author{
M. S. PEREIRA ${ }^{1}$, L. E. P. BORGES ${ }^{2}$ e N.T. MACHADO ${ }^{3}$ \\ ${ }^{1}$ Universidade Federal do Pará, Pós-graduação em Engenharia de Recursos Naturais da Amazônia \\ ${ }^{2}$ Instituto Militar de Engenharia , Seção de Química \\ ${ }^{3}$ Universidade Federal do Pará, Faculdade de Engenharia Química \\ E-mail para contato: marsytonight@gmail.com
}

\begin{abstract}
RESUMO - A ampla utilização dos plásticos gera uma grande quantidade de resíduos que devem ser descartados ou, preferencialmente, reciclados de forma adequada. $\mathrm{O}$ presente trabalho investigou, em escala de laboratório, o processo de craqueamento de polietileno de baixa densidade (PEBD) virgem e de polietileno de alta densidade (PEAD) proveniente de sacolas plásticas de supermercados, focando a produção de frações líquidas semelhantes aos derivados de petróleo. Os testes foram realizados na ausência e na presença de catalisadores; foram testados dois tipos de catalisadores: um ácido (catalisador comercial de FCC) e o outro básico (carbonato de sódio, $\mathrm{Na}_{2} \mathrm{CO}_{3}$ ) em diferentes temperaturas na faixa de 420 a $460{ }^{\circ} \mathrm{C}$. Os produtos líquidos craqueados foram caracterizados por cromatografia em fase gasosa acoplada a espectrometria de massas (CG/EM), espectrometria de infravermelho e espectrometria de ressonância magnética nuclear. Os catalisadores também foram caracterizados por FTIR e difração de raios-X (DRX). Com objetivo de se obter cadeias mais curtas foram realizados alguns testes preliminares em 2 etapas de craqueamento. As análises de cromatografia confirmaram a produção de hidrocarbonetos nas faixas da gasolina, querosene e óleo diesel, com rendimentos acima de $70 \%$. Assim, pode-se demonstrar que o craqueamento de polímeros é uma alternativa tecnológica interessante para o reaproveitamento de resíduos plásticos.
\end{abstract}

\section{INTRODUÇÃO}

Em decorrência do aumento na produção de polímeros, é crescente a preocupação ambiental decorrentes de seu descarte na natureza. Devido seu longo tempo de degradação, estudos relacionados ao desenvolvimento de tecnologias visando à reciclagem de plásticos pós-consumo tem merecido especial atenção. A reciclagem torna-se um elo importante na cadeia de produção dos plásticos, tendo em vista que o seu descarte de forma incorreta é mais criticado pela sociedade do que a poluição gerada durante o processo de produção (AWARA, 2005). Para o sucesso no desenvolvimento de metodologias para reciclagem dos plásticos, é imprescindível investimento em ações de conscientização quanto ao consumo e descarte de forma adequada dos materiais plásticos (ALSALEM, 2009).

No processo de craqueamento, os de polímeros são transformados em moléculas de estrutura 


\section{9 a 22 de outubro de 2014 \\ Florianópolis/SC}

molecular simples, constituídas de carbono e hidrogênio, semelhantes aos hidrocarbonetos e olefinas, produzindo diesel e gasolina através da adição intensa de energia térmica, na presença ou não de catalisadores. A faixa de temperatura para a quebra das cadeias poliméricas pode variar entre $350 \mathrm{e}$ $500{ }^{\circ} \mathrm{C}$. Os catalisadores são utilizados visando à redução da temperatura de craqueamento e a formação de produtos de elevado valor de mercado (MARCILLA, 2007). Variados catalisadores têm sido empregados no processo de degradação catalítica dos plásticos, como: mordenita, FCC, USY, ZSM-5, H-beta, H-Y, SAPO-37, MCM-41 (MARCILLA, 2006). Estes catalisadores mostram diferentes atividades de acordo com a estrutura dos poros, acidez e tamanho do cristal.

Este trabalho teve como objetivo estudar o craqueamento térmico e catalítico do polietileno de baixa densidade (PEBD) virgem e polietileno de alta densidade (PEAD) proveniente de sacolas plásticas de supermercados para obtenção de produtos na faixa do diesel e gasolina, além de hidrocarbonetos leves, como propeno e butenos.

\section{MATERIAS E METÓDOS}

\subsection{Polímeros}

Os experimentos foram realizados com PEBD (polietileno de baixa densidade) cedido pelo Instituto de Macromoléculas (IMA- UFRJ) e com PEAD (polietileno de alta densidade) proveniente de sacolas plásticas de supermercados calcinadas a $200^{\circ} \mathrm{C}$ em 43 minutos com taxa de aquecimento de $3^{\circ} \mathrm{C} / \mathrm{min}$ para diminuir o seu volume.

\subsection{Catalisadores}

Carbonato de sódio $\left(\mathrm{Na}_{2} \mathrm{CO}_{3}\right)$, B. Herzog (PA) foi fornecido pela B. Herzog. O catalisador comercial de FCC foi cedido pela Fábrica Carioca de Catalisadores-FCC S.A.

\subsection{Processo de craqueamento térmico e térmico catalítico}

O craqueamento térmico e termocatalítico foram realizados em um reator de vidro de $100 \mathrm{ml}$, com diâmetro de $3,5 \mathrm{~cm}$ e comprimento de $15 \mathrm{~cm}$, em diferentes temperaturas na faixa de 420 a $460^{\circ} \mathrm{C}$. O reator foi aquecido por um forno cilíndrico posicionado sobre uma placa de agitação magnética. A temperatura dos testes e as taxas de aquecimento eram controladas por um controlador/programador (THERMA, Modelo: TH90DP202-000) ligado a um termopar do tipo K (Ecil, Modelo: QK. 2 ) posicionado junto à parede do reator. A Figura 1 apresenta um esquema simplificado do aparato experimental.

No craqueamento termocatalítico foram utilizados 5 a $10 \%$ em peso, de carbonato de sódio $\left(\mathrm{Na}_{2} \mathrm{CO}_{3}\right)$ e FCC. $\mathrm{O}$ catalisador básico carbonato de sódio $\left(\mathrm{Na}_{2} \mathrm{CO}_{3}\right)$ foi calcinado a $300{ }^{\circ} \mathrm{C}$ por duas horas e o catalisador ácido de FCC foi secado em uma estufa a $80{ }^{\circ} \mathrm{C}$ por 24 horas. A caracterização química dos catalisadores foi realizada através de FTIR E DRX. Utilizou-se $30 \mathrm{~g}$ da massa total do reator de vidro, adicionando-se massa de catalisador prevista para o teste. As taxas de aquecimento foram de 5 e $10^{\circ} \mathrm{C} / \mathrm{min}$ e fluxo de Nitrogênio $\left(\mathrm{N}_{2}\right)$ de $0,4 \mathrm{Nl} / \mathrm{mim}$ foi utilizado em todos os testes. Os 
vapores da reação eram condensados em frasco imerso em banho de gelo. Com objetivo de formar cadeias menores, dois testes (experimentos 9 e 10) foram realizados em 2 etapas: uma primeira etapa térmica e a segunda catalítica utilizando como carga o produto craqueado da primeira reação.

Os produtos líquidos foram armazenados em frascos fechados para posteriores análises de espectroscopia de infravermelho com transformada de Fourier (FTIR), espectroscopia de ressonância magnética nuclear (RMN) e cromatografia gasosa acoplada em à espectrometria de massas (CG/EM).

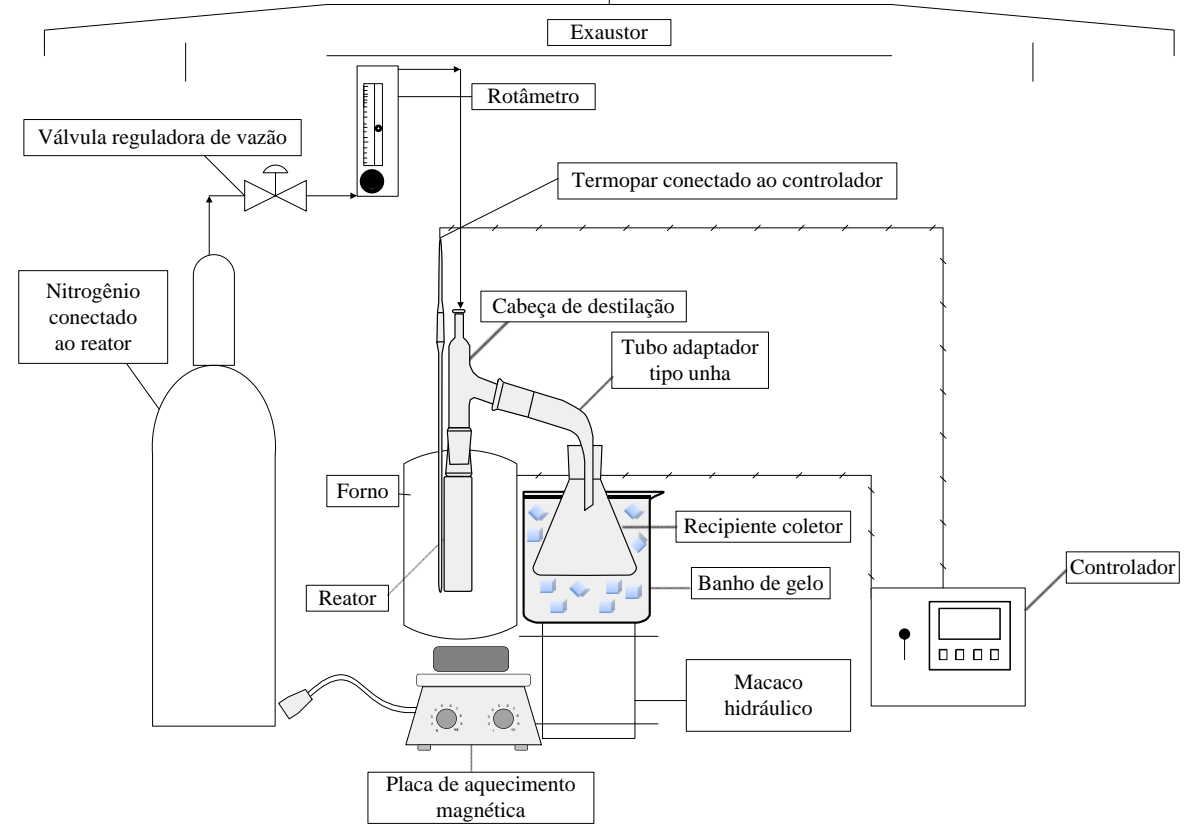

Figura 1 - Aparato experimental utilizado nos testes de craqueamento.

\section{RESULTADOS E DISCUSÕES}

\subsection{Caracterização dos catalisadores}

No espectro de infravermelho, a amostra de carbonato de sódio apresentou as bandas de absorção das vibrações do ânion $\mathrm{CO}_{3}{ }^{2-}$, compreendidos entre $1800-400 \mathrm{~cm}^{-1}$, característicos de carbonatos de metais alcalinos. No carbonato de sódio as bandas de $1446,61 \mathrm{~cm}^{-1}, 879,54 \mathrm{~cm}^{-1} \mathrm{e}$ $702,09 \mathrm{~cm}^{-1}$ são referentes ao estiramento de vibração do $\left(\mathrm{CO}_{3}\right)^{2-}$ fora do plano.

O difratograma da amostra de carbonato de sódio apresentou somente parâmetros de rede de sistema monoclínico, identificado pela ficha da Joint Committee on Powder Diffraction Standards (JCPDS) 77-2082, com algumas raias correspondentes aos planos cristalinos (310) $(2 \theta=35,2),(112)$ $(2 \theta=37,96),(002)(2 \theta=30,08)$ e $(221)(2 \theta=41,27)$. Dados semelhantes foram observados por Da 
SILVA (2010). O catalisador de FCC também foi submetido à análise por difração de raios X apresentando sinais relativos a zeólita HY e a alumina. O catalisador de FCC também foi submetido à análise por difração de raios $\mathrm{X}$ apresentando sinais relativos a zeólita $\mathrm{HY}$ e a alumina, sendo os principais sinais obtidos em $2 \theta \approx 15^{\circ}, 19^{\circ}, 21^{\circ}, 25,5^{\circ}, 38^{\circ}, 52,8^{\circ} \mathrm{C}$ e $66,7^{\circ}$ (RATTON, 2012 e PEDROSA et al , 2005). Segundo RATTON (2012), a área específica $\left(\mathrm{S}_{\mathrm{BET}}\right)$ da amostra foi de $284 \mathrm{~m}^{2} / \mathrm{g}$, apresentando valor de área superficial intermediário entre os valores normalmente observados para a HY e a alumina.

\subsection{Análises químicas dos produtos poliméricos}

A tabela 1 apresenta o rendimento mássico dos produtos de craqueamento das amostras craqueadas sem catalisador, nas temperaturas de 420,440 e $460{ }^{\circ} \mathrm{C}$. A quantidade de produto líquido, foco deste trabalho, aumenta significativamente com o aumento da temperatura de craqueamento. Rendimentos da ordem de $80 \%$ em massa foram obtidos tanto com a amostra de PEBD virgem, como com a amostra de PEAD das sacolas plásticas.

A utilização de resíduos comerciais, como as sacolas plásticas, leva à obtenção de uma quantidade importante de resíduo sólido da reação, mesmo a temperaturas mais altas, provavelmente em função de pigmentos inorgânicos e outros aditivos presentes nesse tipo de material. Não houve formação de resíduo sólido no teste com material virgem a $460{ }^{\circ} \mathrm{C}$.

Tabela 1 - Rendimentos (\% em massa) dos testes de craqueamento térmico de polietileno

\begin{tabular}{|c|c|c|c|c|c|}
\hline \multirow{2}{*}{ Polietileno* } & \multirow{2}{*}{ Teste } & \multirow{2}{*}{ Temperatura $\left({ }^{\circ} \mathrm{C}\right)$} & \multicolumn{3}{|c|}{ Balanço de massas $(\%$ peso) } \\
\cline { 3 - 6 } & 1 & Resíduo & Produtos & Gases \\
\hline \multirow{3}{*}{ PEBD virgem } & 2 & $420^{\circ} \mathrm{C}$ & 79,4 & 13,2 & 7,4 \\
\cline { 2 - 6 } & 3 & $440^{\circ} \mathrm{C}$ & 47,7 & 43,8 & 8,5 \\
\hline PEAD sacolas & 4 & $460^{\circ} \mathrm{C}$ & 0,0 & 88,3 & 11,7 \\
\hline
\end{tabular}

A Tabela 2 apresenta os rendimentos dos produtos obtidos na presença de catalisador nas temperaturas de 440 e $460{ }^{\circ} \mathrm{C}$. Novamente, os rendimentos em produto liquido foram acima de $70 \%$ nos testes realizados a $460{ }^{\circ} \mathrm{C}$, sendo o melhor rendimento obtido com o carbonato de sódio. $\mathrm{O}$ teste com $5 \%$ de carbonato de sódio a $440{ }^{\circ} \mathrm{C}$ apresentou um resultado bem inferior, provavelmente mais pelo fator temperatura que pelo fator catalisador. No caso das amostras de sacolas plásticas, os catalisadores apresentaram rendimentos em produto líquido semelhantes, com uma ligeira vantagem para o catalisador de FCC. 
Tabela 2 - Rendimentos (\% em massa) dos testes de craqueamento termocatalítico de polietileno a

\begin{tabular}{|c|c|c|c|c|c|}
\hline \multirow{2}{*}{ Polietileno* } & \multirow{2}{*}{ Teste } & \multirow{2}{*}{ Catalisador $(\%)$} & \multicolumn{3}{|c|}{ Balanço de massas (\% peso) } \\
\cline { 3 - 5 } & & & Resíduo & Produtos & Gases \\
\hline \multirow{2}{*}{ PEBD virgem } & $5^{*}$ & $5 \% \mathrm{Na}_{2} \mathrm{CO}_{3}$ & 46,9 & 44,9 & 8,2 \\
\cline { 2 - 5 } & 6 & $10 \% \mathrm{Na}_{2} \mathrm{CO}_{3}$ & 0,0 & 90,6 & 9,4 \\
\hline \multirow{2}{*}{ PEAD sacolas } & 7 & $10 \% \mathrm{Na}_{2} \mathrm{CO}_{3}$ & 13,6 & 68,4 & 18,0 \\
\cline { 2 - 5 } & 8 & $10 \% \mathrm{FCC}$ & 8,1 & 76,0 & 15,9 \\
\hline
\end{tabular}

*Teste realizado a $440^{\circ} \mathrm{C}$

A Tabela 3 apresenta os rendimentos dos produtos do craqueamento em duas etapas, utilizando a temperatura de $460^{\circ} \mathrm{C}$ nas duas etapas. Estes testes foram realizados para se verificar a possibilidade de redução do tamanho das cadeias moleculares por um segundo craqueamento, visando a produção de misturas mais leves, de maior valor agregado. Em termos de rendimento, o re-craqueamento leva a uma elevada produção de produtos líquidos, sem a formação de resíduo. Não se observou diferença de rendimento entre os catalisadores usados.

Tabela 3 - Rendimentos (\% em massa) dos testes de craqueamento termocatalítico de polietileno em duas etapas, a $460{ }^{\circ} \mathrm{C}$

\begin{tabular}{|c|c|c|c|c|c|}
\hline \multirow{2}{*}{ Polietileno* } & \multirow{2}{*}{ Teste } & \multirow{2}{*}{ Catalisador $(\%)$} & \multicolumn{3}{|c|}{ Balanço de massas (\% peso) } \\
\cline { 3 - 5 } & & Resíduo & Produtos & Gases \\
\hline \multirow{3}{*}{ PEAD sacolas } & \multirow{2}{*}{9} & Térmico & 23,1 & 66,8 & 10,1 \\
\cline { 3 - 5 } & \multirow{2}{*}{10} & $10 \% \mathrm{Na}_{2} \mathrm{CO}_{3}$ & 0,0 & 93,1 & 6,9 \\
\cline { 3 - 5 } & & Térmico & 17,9 & 72,9 & 9,2 \\
\cline { 3 - 5 } & & $10 \% \mathrm{FCC}$ & 0,0 & 94,9 & 5,1 \\
\hline
\end{tabular}

\subsection{Análises químicas dos produtos poliméricos}

As principais vibrações observadas nos espectros de infravermelhos dos produtos líquidos estão apresentadas na Tabela 4. De uma forma geral, os espectros apresentam vibrações típicas de misturas de hidrocarbonetos, com poucas diferenças entre os produtos. Bandas de forte absorção foram observadas entre 3000 a $2800 \mathrm{~cm}^{-1}$, correspondentes ao estiramento vibracional $\mathrm{C}-\mathrm{H}$ dos grupos $\mathrm{CH}_{2} \mathrm{e}-\mathrm{CH}_{3}$. Observou-se também em todos os espectros as bandas de absorção em 2924 e $2850 \mathrm{~cm}^{-1}$,

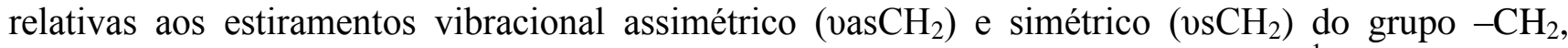

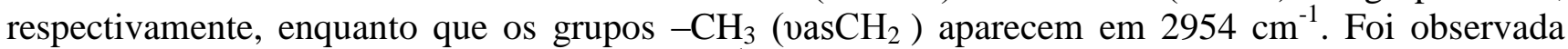
também a presença de banda de 721 e $719 \mathrm{~cm}^{-1}$ característica de produtos que possuem cadeias com mais de 4 carbonos. Vale ainda destacar a presença de estiramentos de deformação axial que evidenciam a presença de olefinas $(\mathrm{C}=\mathrm{C})$ em todos os produtos, caracterizados pelas vibrações em 3076 e $1641 \mathrm{~cm}^{-1}$. Constataram-se ainda as bandas 991 e $908 \mathrm{~cm}^{-1}$ relativas a alquenos monosubstituídos $\left(\mathrm{RCH}=\mathrm{CH}_{2}\right)$. Também foi possível verificar bandas de absorção características da deformação angular simétrica da ligação $\mathrm{C}-\mathrm{H}$ do metileno $\left(\mathrm{CH}_{2}\right)$ em aproximadamente $1462 \mathrm{~cm}^{-1}$ e do 
grupo metila em $1377 \mathrm{~cm}^{-1}$ em todos os espectros de infravermelho.

Tabelas 4 - Principais absorção do FTIR das amostras

\begin{tabular}{|c|c|c|}
\hline Polietileno* & Teste & Principais bandas de absorção no FTIR \\
\hline \multirow{5}{*}{$\begin{array}{l}\text { PEBD } \\
\text { virgem }\end{array}$} & 1 & \multirow{3}{*}{$\begin{array}{l}\text { a) def. assimétrica e simétrica de } \mathrm{CH}_{2}, 2924 \text { e } 2850 \mathrm{~cm}^{-1} \text {; b) } \\
\text { def. assimétrica de } \mathrm{CH}_{3}, 2954 \mathrm{~cm}^{-1} \text {; c) vibração axial C=C, } \\
1641 \mathrm{~cm}^{-1} \text {, d) def. angular simétrica de } \mathrm{CH}_{2} \mathrm{~cm}^{-1}, 1465 ; \text { e) def. } \\
\text { angular simétrica de } \mathrm{CH}_{3}, 1377 \mathrm{~cm}^{-1}\end{array}$} \\
\hline & 2 & \\
\hline & 3 & \\
\hline & 5 & \multirow{2}{*}{$\begin{array}{l}\text { a) def. assimétrica e simétrica de } \mathrm{CH}_{2}, 2922 \text { e } 2850 \mathrm{~cm}^{-1} \text {; } \\
\text { b) def. assimétrica de } \mathrm{CH}_{3}, 2953 \mathrm{~cm}^{-1} \text {; c) vibração axial C=C } \\
1641 \mathrm{~cm}^{-1} \text {; d) def. angular simétrica de } \mathrm{CH}_{2} \mathrm{~cm}^{-1}, 1462 \text {; e) def } \\
\text { angular simétrica de } \mathrm{CH}_{3}, 1377 \mathrm{~cm}^{-1}\end{array}$} \\
\hline & 6 & \\
\hline \multirow{3}{*}{ PEAD } & 4 & $\begin{array}{l}\text { a) def. assimétrica e simétrica de } \mathrm{CH}_{2}, 2922 \text { e } 2850 \mathrm{~cm}^{-1} \text {; } \\
\text { b) def. assimétrica de } \mathrm{CH}_{3}, 2954 \mathrm{~cm}^{-1} \text {; c) vibração axial } \mathrm{C}=\mathrm{C} \text {, } \\
1647 \mathrm{~cm}^{-1}, \text { d) def. angular simétrica de } \mathrm{CH}_{2} \mathrm{~cm}^{-1}, 1465 ; \text { e) def. } \\
\text { angular assimétrica e simétrica de } \mathrm{CH}_{3}, 1458 \text { e } 1375 \mathrm{~cm}^{-1}\end{array}$ \\
\hline & 7 & $\begin{array}{l}\text { a) def. assimétrica e simétrica de } \mathrm{CH}_{2}, 2922 \text { e } 2852 \mathrm{~cm}^{-1} \text {; } \\
\text { b) def. assimétrica de } \mathrm{CH}_{3}, 2954 \mathrm{~cm}^{-1} \text {; c) vibração axial } \mathrm{C}=\mathrm{C} \text {, } \\
1641 \mathrm{~cm}^{-1} \text {, d) def. angular simétrica de } \mathrm{CH}_{2}, 1463 \mathrm{~cm}^{-1} \text {; e) def. } \\
\text { angular assimétrica e simétrica de } \mathrm{CH}_{3}, 1458 \text { e } 1377 \mathrm{~cm}^{-1}\end{array}$ \\
\hline & 8 & $\begin{array}{l}\text { a) def. assimétrica e simétrica de } \mathrm{CH}_{2}, 2924 \text { e } 2852 \mathrm{~cm}^{-1} \text {; } \\
\text { b) def. assimétrica e simétrica de } \mathrm{CH}_{3}, 2956 \text { e } 2870 \mathrm{~cm}^{-1} \text {; c) } \\
\text { vibração axial C=C, } 1641 \mathrm{~cm}^{-1} \text {, d) def. angular simétrica de } \\
\mathrm{CH}_{2} \mathrm{~cm}^{-1}, 1462 \text {; e) def. angular simétrica de } \mathrm{CH}_{3}, 1377 \mathrm{~cm}^{-1}\end{array}$ \\
\hline \multirow{2}{*}{$\begin{array}{l}\text { PEAD em } \\
\text { duas etapas }\end{array}$} & 9 & $\begin{array}{l}\text { a) def. assimétrica e simétrica de } \mathrm{CH}_{2}, 2924 \text { e } 2852 \mathrm{~cm}^{-1} \text {; } \\
\text { b) def. assimétrica de } \mathrm{CH}_{3}, 2954 \text {; c) vibração axial C=C, } 1641 \\
\mathrm{~cm}^{-1} \text {, d) def. angular simétrica de } \mathrm{CH}_{2} \mathrm{~cm}^{-1}, 1465 \text {; e) def. } \\
\text { angular simétrica de } \mathrm{CH}_{3}, 1377 \mathrm{~cm}^{-1}\end{array}$ \\
\hline & 10 & $\begin{array}{l}\text { a) def. assimétrica e simétrica de } \mathrm{CH}_{2}, 2922 \text { e } 2852 \mathrm{~cm}^{-1} \text {; } \\
\text { b) def. assimétrica de } \mathrm{CH}_{3}, 2954 \text {; c) vibração axial C=C, } 1641 \\
\mathrm{~cm}^{-1} \text {, d) def. angular simétrica de } \mathrm{CH}_{2} \mathrm{~cm}^{-1}, 1463 \text {; e) def. } \\
\text { angular simétrica de } \mathrm{CH}_{3}, 1377 \mathrm{~cm}^{-1}\end{array}$ \\
\hline
\end{tabular}

Os deslocamentos químicos dos espectros de Ressonância Magnética Nuclear (RMN) de ${ }^{13} \mathrm{C}$ e ${ }^{1} \mathrm{H}$ do produto líquido do craqueamento do polietileno são apresentados nas Tabelas 5 e 6. 
Tabela 5 - Deslocamentos químicos de ${ }^{13} \mathrm{C}$ dos experimentos: 2(térmico), experimento 9(duas etapas com $10 \% \mathrm{Na}_{2} \mathrm{CO}_{3}$ ) e experimento 10 (duas etapas com $10 \%$ FCC)

\begin{tabular}{|c|c|c|c|}
\hline Polietileno* & Testes & Tipos de carbono ${ }^{13} \mathrm{C}$ & Deslocamento químico (ppm) \\
\hline PEBD virgem & 2 & $\begin{array}{c}\text { Hidrocarbonetos }\left(\mathrm{CH}_{2} \text { e } \mathrm{CH}_{3}\right) \\
\text { Olefinas }(\mathrm{C}=\mathrm{C})\end{array}$ & $\begin{array}{c}14,08-33,81 \\
114,02 \text { e } 139,21\end{array}$ \\
\hline \multirow{2}{*}{$\begin{array}{c}\text { PEAD } \\
\text { sacolas,em } \\
\text { duas etapas }\end{array}$} & 9 & $\begin{array}{c}\text { Hidrocarbonetos }\left(\mathrm{CH}_{2} \text { e } \mathrm{CH}_{3}\right) \\
\text { Olefinas }(\mathrm{C}=\mathrm{C})\end{array}$ & $14,08-33,81$ \\
\cline { 2 - 4 } & 10 & $\begin{array}{c}\text { Hidrocarbonetos }\left(\mathrm{CH}_{2} \text { e } \mathrm{CH}_{3}\right) \\
\text { Olefinas }(\mathrm{C}=\mathrm{C})\end{array}$ & 114,02 e 139,21 \\
\hline
\end{tabular}

Tabela 6 - Deslocamentos químicos de ${ }^{1} \mathrm{H}$ dos experimentos: 2 (térmico),experimento 9(duas etapas com $10 \% \mathrm{Na}_{2} \mathrm{CO}_{3}$ ) e experimento 10 (duas etapas com $10 \% \mathrm{FCC}$ )

\begin{tabular}{|c|c|c|c|}
\hline Polietileno* & Experimentos & Tipos de protón ${ }^{1} \mathrm{H}$ & Deslocamento químico (ppm) \\
\hline PEBD & & Alquila primária & $0,88-0,92$ \\
VIRGEM & 2 & Alquila secundária & 1,27 \\
& & Olefinas & $4,70-5,83$ \\
\hline & \multirow{2}{*}{9} & Alquila primária & $0,88-0,88$ \\
PEAD em & & Alquila secundária & 1,25 \\
\cline { 2 - 4 } duas etapas & \multirow{2}{*}{10} & Olefinas & $4,91-5,00$ \\
& & Alquila primária & $0,90-0,92$ \\
& & Alquila secundária & 1,29 \\
& & Olefinas & $4,94-5,03$ \\
\hline
\end{tabular}

Todos os espectros de RMN tanto de ${ }^{13} \mathrm{C}$ e ${ }^{1} \mathrm{H}$ dos produtos líquidos do craqueamento do polietileno, tanto catalíticos como térmico, são semelhantes e forneceram basicamente as mesmas informações para todas as amostras. São espectros típicos de hidrocarbonetos de cadeias longas com algumas insaturações. Este resultado corrobora aquilo que já tinha sido indicado pela analise de FTIR.

Os produtos líquidos das reações de duas etapas foram submetidos a análise por cromatografia gasosa acoplada a espectrômetro de massas. Do ponto de vista qualitativo, as análises dos testes 9 e 10 são muito semelhantes, revelando a presença de hidrocarbonetos saturados e $\alpha$ mono-insaturados lineares e de cadeias longas. $\mathrm{Na}$ análise de massas, foram identificadas, com $95 \%$ de probabilidade, séries homologas de hidrocarbonetos saturados e a mono-insaturados de 7 a 26 carbonos. Infelizmente, não foi possível observar uma variação significativa entre os produtos da primeira etapa (só térmica) e os da segunda etapa (catalítica). É possível que, devido a configuração da unidade e ao procedimento experimental, a carga da segunda etapa tenha sido basicamente vaporizada, sem ter tido tempo de contato suficiente com os catalisadores para que se tivesse uma alteração na composição dos produtos. 


\section{CONCLUSÃO}

Os testes de craqueamento térmico e termocatalítico de amostras de polietileno indicaram claramente a viabilidade da transformação de resíduos plásticos em produtos líquidos similares aos derivados de petróleo. O uso de materiais residuais, como sacolas plásticas usadas, aumenta a formação de resíduo sólido na reação. A temperatura de $460{ }^{\circ} \mathrm{C}$ foi necessária para se obter bons rendimentos, tanto nos testes térmicos como nos catalíticos. Não se observou diferenças muito significativas entre os produtos obtidos com os catalisadores ácido e básico. Da mesma forma, os testes em duas etapas não forneceram resultados diferentes. Novos testes serão realizados com catalisadores mais ácidos.

\section{REFERÊNCIAS}

AL-SALEM, S.M., LETTIERI, P and BAEYENS, J. Recycling and recovery routs of plastics solid wastes (PSW): A review. Waste Management 29 (2009) 2625-2643.

AWARA, F. and PAVEL, D. Review: Recycling of PET. European Polymer Journal 41 (2005) 1453-1477.

Da SILVA, R.M., Craqueamento termocatalítico de óleos vegetais e gorduras, Tese de Doutorado, Instituto Militar de Engenharia, Rio de Janeiro, 2010.

HU, Y., MA, Y., ZHOU, Y., NIE, F., DUAN, X., PEI, C. Hen eggwhite mediated stack crystallization of calcium carbonate. Journal of Crystal Growth 2010:312:831-836.

MARCILLA, A., GÓMES-SIURANA, A., BERENGUER, D. Study of the influence of the characteristics of different acid solids in the catalytic pyrolysis of different polymers. Applied Catalysis A: General 301 (2006) 222-231.

MARCILLA, A., BELTRÁN, M.I., SIURANA, A., NAVARRO, R., and VALDÉS, F. A global kinetic model as a tool to reproduce the deactivation behaviour of the HZSM-5 zeolite in the catalytic ctacking of low-density polyethylene. Applied Catalysis A: General 328 (2007) $124-131$.

PEDROSA,A.M.G.,SOUZA,M.J.B.,MELO,D.M.A. and ARAUJO,A.S. Cobalt and nickel supported on HY zeolite: Synthesis,characterization and catalytic properties. Materials Research Bulletin 41 (2006) 1105-1111.

RATTON, A. R., Produção de Hidrocarbonetos a partir do Craqueamento de Resíduos provenientes de Caixa de Gordura, Dissertação de Mestrado, Instituto Militar de Engenharia,Rio de Janeiro, 2012. 